\title{
Probing the dust formation zone in IRC +10216 with the SMA
}

\author{
Nimesh A. Patel \\ Harvard-Smithsonian Center for Astrophysics \\ E-mail: npatel@cfa.harvard.edu
}

\section{Carl Gottlieb}

Harvard-Smithsonian Center for Astrophysics

E-mail: cgottlieb@cfa.harvard.edu

\section{Ken Young}

Harvard-Smithsonian Center for Astrophysics

E-mail: kyoung@cfa.harvard.edu

Submillimeter imaging of high-excitation molecular rotational lines provides a powerful probe of the dust formation and acceleration region $\left(R \leq 5 \times 10^{16} \mathrm{~cm}\right)$ in the extreme carbon-rich star IRC +10216 . We present Submillimeter Array (SMA) observations of several rotational lines of SiCC from our 345 and $400 \mathrm{GHz}$ spectral-line surveys, showing a progression from rectangular to narrow triangular line-profiles, and spatially extended to point-like unresolved emission, as a function of increasing energy $E_{\mathrm{u}}$. Maps of $\mathrm{SiC}$ and $\mathrm{SiN}$ emission show asymmetrical extended shell-like structures. These results are compared with published chemical model calculations of the radial abundances of molecules believed to be relevant for dust formation.

The Life Cycle of Dust in the Universe: Observations, Theory, and Laboratory Experiments - LCDU 2013, 18-22 November 2013

Taipei, Taiwan 


\section{Introduction \& motivation}

Interstellar dust grains are thought to evolve in the colder regions of space from the gas and solids present in the outflows from Asymptotic Giant Branch (AGB) stars. IRC+10216 is an ideal source to study the physical and chemical processes that occur in the circumstellar envelopes of carbon-rich AGB stars, because it is close to the Sun $(\sim 150 \mathrm{pc})$, the mass loss is high, and it is very rich in molecules. Spectral line-surveys allow us to identify new molecules, and from multiple transitions of the same molecule, we can also infer the physical conditions of the circumstellar gas. Direct observation of small metal bearing molecules in the inner envelope of AGB stars is becoming feasible with sensitive interferometers in the submillimeter band [1,2]. These recent studies show that higher excitation submillimeter wavelength rotational lines are powerful probes, particularly with interferometry, of the inner envelope of IRC+10216 in the regions of dust formation and acceleration of the outflow.

\section{Silicon carbide and silicon nitride in IRC $+\mathbf{1 0 2 1 6}$}

Silicon carbide is a well known constituent of solid particles in circumstellar envelopes. Presolar $\mathrm{SiC}$ grains found in meteorites are believed to have originated in carbon stars [3, 4]. Chemical equilibrium calculations on the production of these molecules have been carried out in the last few decades [5] and also more recently [6,7]. But very little is known from observations about the spatial distribution of $\mathrm{SiC}$ in gas phase.

Two successive rotational transitions of $\mathrm{SiC}$ at 315 and $354 \mathrm{GHz}$ were observed in the ground ${ }^{3} \Pi_{2}$ fine structure component with the SMA [1]. Prior to this, lines of gaseous $\mathrm{SiC}$ in the ${ }^{3} \Pi$ ground electronic state were observed in the $157-283 \mathrm{GHz}$ range with single antennas $[8,9,10]$, at low angular resolution of typically $10^{\prime \prime}-30^{\prime \prime}$. Figure 1 shows the $\mathrm{SiC}$ spectrum and map of the $315 \mathrm{GHz}$ line observed with the SMA at $3^{\prime \prime}$ resolution. In spectra observed both with single antennas and the SMA, the line profiles are very narrow with distinct horns, where we find that the FWHM $\sim 2.5 \mathrm{~km}$ $\mathrm{s}^{-1}$ and $V_{\exp }=12.8 \mathrm{~km} \mathrm{~s}^{-1}$ is close to the terminal velocity. The flux observed with the SMA (1.2 Jy km s$~^{-1}$ at $315 \mathrm{GHz}$, and $1.4 \mathrm{Jy} \mathrm{km} \mathrm{s}^{-1}$ at $354 \mathrm{GHz}$ ) is comparable to that observed in transitions at a slightly lower frequency of $275.7 \mathrm{GHz}$ with the $10 \mathrm{~m}$ ARO antenna (27" diameter beam [10]). Although the flux is not very high, there is no evidence for $\mathrm{SiC}$ in the dust formation zone within a few arcsec of the star as Figure 1 shows. Cernicharo et al. [8] concluded on the basis of the line profile shapes that $\mathrm{SiC}$ is confined to the outer envelope with an estimated column density of $6 \times 10^{13} \mathrm{~cm}^{-2}$ averaged over their $17^{\prime \prime}$ diameter antenna beam. Our derived column density of $2.4 \times 10^{13} \mathrm{~cm}^{-2}$ is comparable to this original estimate, but the fractional abundance (w.r.t. $\mathrm{H}_{2}$ ) of $1.5 \times 10^{-9}$ is significantly higher than chemical model predictions [6] and the calculated radial distribution of $\mathrm{SiC}$ [11] is not consistent with our measurements which establish that the gaseous $\mathrm{SiC}$ is about ten times closer to the star. However, similar maps of lower excitation lines are needed to confirm this. Results for SiN are similar, as shown in Figure 2. The channel maps confirm that the emission arises from an expanding shell. We estimate the column density for $\operatorname{SiN} \sim 3.2 \times 10^{13}$ $\mathrm{cm}^{-2}$ and fractional abundance of $2.0 \times 10^{-9}$, with $T_{\mathrm{ex}} \sim 40 \mathrm{~K}$. Our SiN column density is in good agreement with the value quoted by Turner in his detection paper [12]. 

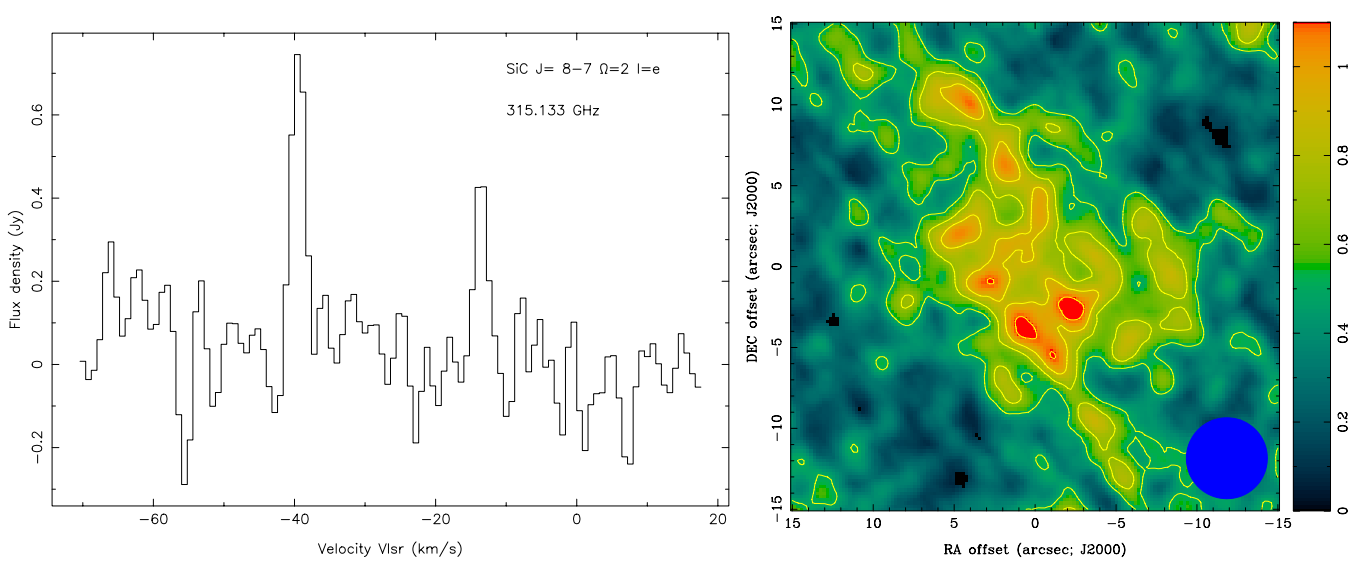

Figure 1: Spectrum and map of integrated emission of $\mathrm{SiC}$ near $315 \mathrm{GHz}$. The map suggests that (1) $\mathrm{SiC}$ is localized in an asymmetrical extended shell-like structure with an approximate radius from the star of about $4^{\prime \prime}$, and (2) there is no evidence for $\mathrm{SiC}$ within the dust condensation radius of $0.1^{\prime \prime}$.
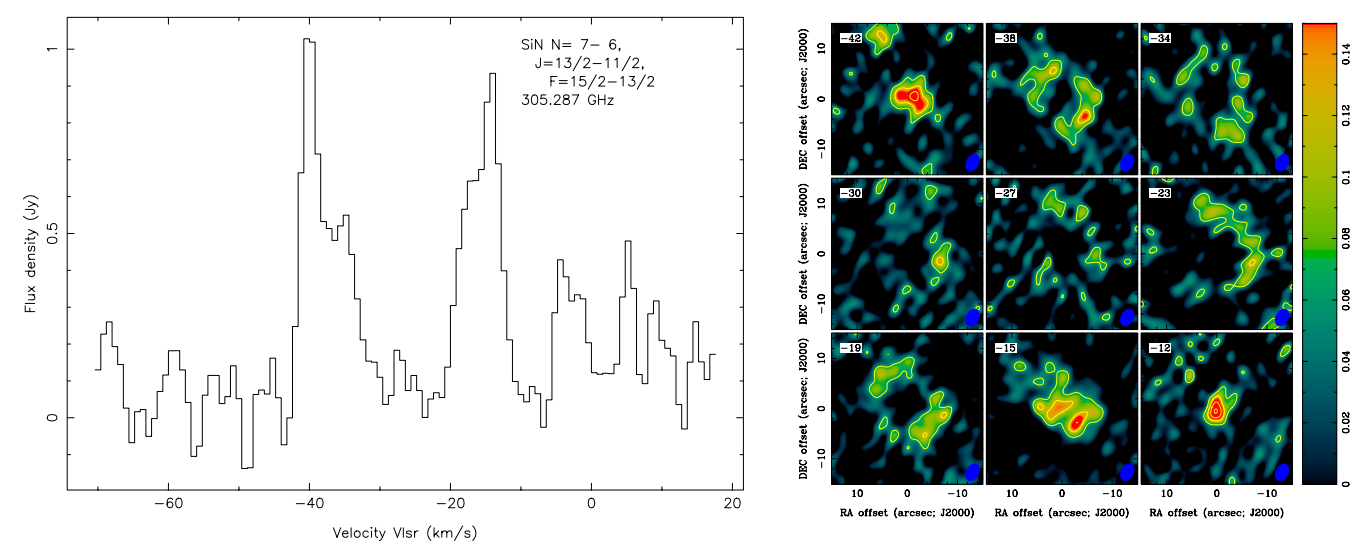

Figure 2: Spectrum and channel map of SiN emission near $305 \mathrm{GHz}$.

In the past couple of years, we have measured many lines of SiCC in IRC+10216 in the $345 \mathrm{GHz}$ and $400 \mathrm{GHz}$ bands at high angular resolution with the SMA [1, 13]. Of the 4 silicon carbides observed with radio telescopes ( $\mathrm{SiC}, \mathrm{SiCC}$, cyclic $\mathrm{SiC}_{3}$, and $\mathrm{SiC}_{4}$ ), only $\mathrm{SiCC}$ is observed in the inner envelope of IRC+10216 (the other 3 are in shells $\geq 4^{\prime \prime}$ from the star). The measured line widths confirm that we are directly observing SiCC in the dust condensation region close to the star. The line profiles change from rectangular to narrow triangular shape with increasing $E_{\mathrm{u}}$. The expansion velocities derived from the line widths are plotted vs $E_{\mathrm{u}}$ in Figure 3, showing a transition at $\sim 500 \mathrm{~K}$.

\section{Salts and metal halides}

Spectra and maps of salts $\mathrm{NaCl}$ and $\mathrm{KCl}$ at $\sim 351 \mathrm{GHz}$ (not shown here) confirm that the emission appears symmetrical and confined to within a few arcseconds around the star, but some of 

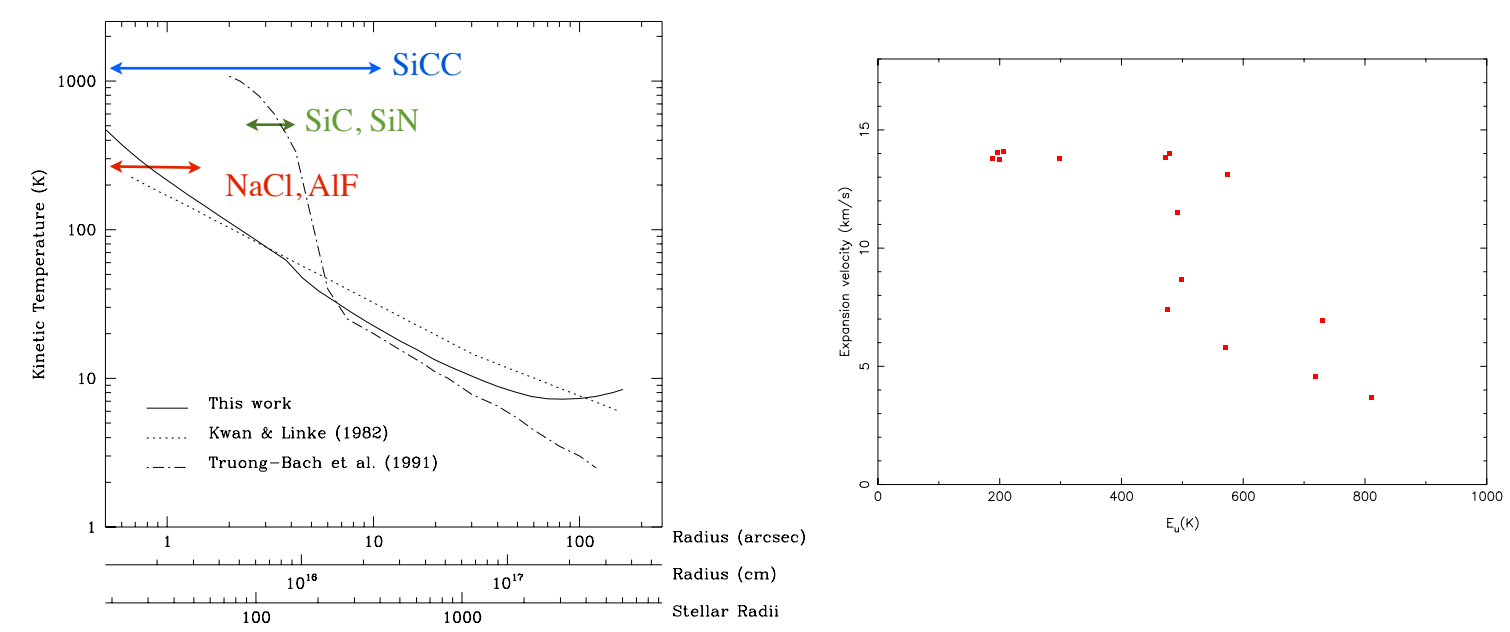

Figure 3: Left: This figure (from [14]) shows the kinetic temperature as a function of radial distance from the star. The radial distribution of various molecules as suggested from SMA results, are shown with colored arrows. Right: Expansion velocity ( $v_{\mathrm{exp}}$ ) versus $E_{\mathrm{u}}$ derived from measured line profiles of SiCC in the $345 \mathrm{GHz}$ and $400 \mathrm{GHz}$ bands. For $E_{\mathrm{u}}<400 \mathrm{~K}, v_{\exp }$ is comparable to the terminal velocity of $14.5 \mathrm{~km} \mathrm{~s}^{-1}$, but $v_{\exp }$ decreases at higher $E_{\mathrm{u}}$ approaching $5 \mathrm{~km} \mathrm{~s}^{-1}$ (the velocity in the dust condensation region) for $E_{\mathrm{u}}=700-800 \mathrm{~K}$.

the gas reaches the terminal velocity. $\mathrm{AlF}$ and $\mathrm{AlCl}$ (near $296 \mathrm{GHz}$ ) show two spatial components - a spatially unresolved, and a very compact shell just barely resolved with our $3^{\prime \prime}$ beam.

\section{References}

[1] N. A. Patel, K. H. Young, C. A. Gottlieb, et al. 2011, ApJS, 191, 17

[2] J. Cernicharo, F. Daniel, A. Castro-Carrizo, et al., 2013, ApJ, 778, L25

[3] D. S. N. Parker, A. V. Wilson, R. I. Kaiser, et al. 2013, ApJ, 770, 33

[4] Y. Yasuda, \& T. Kozasa 2012, ApJ, 745, 159

[5] A. Glassgold, 1996, Annu. Rev. Astron. Astrophys, 34, 241

[6] I. Cherchneff 2012, A\&A, 545, A12

[7] M. Agúndez, J. P. Fonfría, J. Cernicharo, et al. 2012, A\&A, 543, A48

[8] J. Cernicharo, C. Gottlieb, M. Guélin, et al. 1989, ApJ, 341, L25

[9] J. Cernicharo, M. Guélin, C. Kahane, 2000, A\&AS, 142,181

[10] E. Tenenbaum, J. Dodd, S. Milam, et al., 2010, ApJ, 720, 102

[11] D. MacKay \& S. Charnley, 1999, MNRAS, 302,793

[12] B. Turner, 1992, ApJ, 388, L35

[13] N. A. Patel, K. H. Young, S. Brünken, et al. 2009, ApJ, 692, 1205

[14] M. Crosas \& K. Menten, 1997, ApJ, 483, 913 